\title{
A THEOREM ON CONJUGATE NETS IN PROJECTIVE HYPERSPACE
}

\author{
P. O. BELL
}

A theorem proved by C. C. Hsiung in a recent paper [1 ${ }^{1}$ may be stated as follows: In a linear space $S_{n}$ of $n(\geqq 3)$ dimensions let $N_{x}$ be a conjugate net and $\pi$ be a fixed hyperplane; then the points $M, \bar{M}$ of intersection of the fixed hyperplane $\pi$ and the two tangents at a point $x$ of the net $N_{x}$ describe two conjugate nets $N_{M}, N_{\bar{M}}$ in the hyperplane $\pi$, respectively, and one of the two nets $N_{M}, N_{\bar{M}}$ is a Laplace transformed net of the other. The purpose of this note is to prove, in an elementary manner, a general theorem of which the above stated theorem of Hsiung is a specialization. The statement of the theorem follows:

In a linear space $S_{n}$ of $n(\geqq 3)$ dimensions let $N_{x}$ be a conjugate (parametric) net. Let $M, \bar{M}$ be points on the $u$-, v-tangents at $x$ of the net $N_{x}$, respectively, which describe two nets $N_{M}, N_{\bar{M}}$ having the property that the tangent plane of $N_{M}\left(N_{\bar{M}}\right)$ at $M(\bar{M})$ passes through $\bar{M}(M)$. The nets $N_{M}, N_{\bar{M}}$ are conjugate nets and each one of them is a Laplace transformed net of the other one.

For the proof let us observe first that since $N_{x}$ is a conjugate net, the points $M, \bar{M}, \partial \bar{M} / \partial u$, and $\partial M / \partial v$ lie in the tangent plane to $N_{x}$ at $x$; this plane is therefore determined by the points $M, \partial \bar{M} / \partial u$, $\partial M / \partial v$. The conditions that the tangent planes to $N_{M}$ and $N_{\bar{M}}$ at $M$ and $\bar{M}$, respectively, pass through the points $\bar{M}$ and $M$ are equivalent to the conditions that the matrices

$$
\left(M, \frac{\partial M}{\partial u}, \frac{\partial M}{\partial v}, \bar{M}\right), \quad\left(\bar{M}, \frac{\partial \bar{M}}{\partial u}, \frac{\partial \bar{M}}{\partial v}, M\right)
$$

be of rank three. It follows that $\partial \bar{M} / \partial u$ and $\partial M / \partial v$ are expressible by linear relations

$$
\frac{\partial \bar{M}}{\partial u}=a \bar{M}+b M, \quad \frac{\partial M}{\partial v}=\alpha M+\beta \bar{M},
$$

since the tangent planes to $N_{M}$ and $N_{M}$ at $M$ and $\bar{M}$ cannot coincide with the tangent plane to $N_{x}$ at a generic (nonplanar) point $x$ of $N_{x}$. From the form of relations (1) it follows that each of the points $M, \bar{M}$ satisfies an equation of Laplace, and, therefore, each of the nets $N_{M}, N_{\bar{M}}$ is a conjugate net. Furthermore, the point $\bar{M}$ is the

Received by the editors July 28, 1951.

${ }_{1}$ Numbers in brackets refer to the references at the end of the note. 
first Laplace transformed point of $M$ with respect to the net $N_{M}$ and the point $M$ is the minus-first Laplace transformed point of $\bar{M}$ with respect to the net $N_{\bar{M}}$. These additional facts are easily established by use of equations (1) in verifying that $\bar{M}(M)$ is the point on the tangent to the $v(u)$-curve of $N_{M}\left(N_{\bar{M}}\right)$ at $M(\bar{M})$ at which this line touches the edge of regression of the developable surface which it generates as the point $M(\bar{M})$ varies on the $u(v)$-curve of $N_{M}\left(N_{\bar{M}}\right)$. For example, the point on the line $M \bar{M}$ where this line touches the edge of regression of the developable which it generates as $M$ varies over the $u$-curve of $N_{M}$ is the point $\sigma=\bar{M}+\mu M$ in which $\mu$ is determined so that the point $\partial \sigma / \partial u$ lies on the line $M \bar{M}$; that is to say, a linear relation

$$
\frac{\partial \sigma}{\partial u} \equiv \frac{\partial \bar{M}}{\partial u}+\mu \frac{\partial M}{\partial u}+M \frac{\partial \mu}{\partial u}=c M+d \bar{M}
$$

must be fulfilled. On substituting in (2) for $\partial \bar{M} / \partial u$ the right member of the first equation of (1) the resulting equation is

$$
a \bar{M}+\left(b+\frac{\partial \mu}{\partial u}\right) M+\mu \frac{\partial M}{\partial u}=c M+d \bar{M} .
$$

Since $\partial M / \partial u$ is linearly independent of $M, \bar{M}$ (the tangents to $N_{M}$ being assumed distinct at $M), \mu$ must vanish. Hence the required point $\sigma$ is found to be the point $\bar{M}$.

To show that the theorem of Hsiung is a specialization of this theorem the procedure is as follows. The intersections of the $u$ - and $v$-tangents to $N_{x}$ at $x$ with the fixed hyperplane are the points $M$ and $\bar{M}$, respectively. Since $N_{x}$ is a conjugate net, the $u$-tangent at $x$ generates a developable as $x$ varies over the $v$-curve. Hence, the tangent line at $M$ to the $v$-curve of $N_{M}$ must lie in the tangent plane to $N_{x}$ at $x$ : that is to say, this tangent line is the line $M \bar{M}$ of intersection of the tangent plane to $N_{x}$ at $x$ and the hyperplane $\pi$. Similarly, the tangent at $\bar{M}$ to the $u$-curve of $N_{\bar{M}}$ passes through $M$. The conditions of the general theorem are therefore satisfied and the conclusion can be drawn.

Hsiung's theorem for $n=3$ is an analogue of a theorem of $\mathrm{B}$. $\mathrm{Su}$ $\left[2\right.$, p. 372] in which the conclusion is the same but the given net $N_{x}$ is assumed to be an asymptotic net. If in the hypothesis of the general theorem proved in this note the net $N_{x}$ is assumed to be an asymptotic net instead of a conjugate net, the same conclusion holds, and the theorem which results is a generalization of the theorem of $\mathrm{Su}$. In the proof of this theorem, the details of which are omitted, the 
points $\partial M / \partial u$ and $\partial \bar{M} / \partial v$ are found to lie on the line $M \bar{M}$ because: (1) the osculating planes of the $u$ - and $v$-curves of the net $N_{x}$ coincide with the tangent plane to $N_{x}$ at $x$, respectively, and (2) the tangent plane at $M(\bar{M})$ to the net $N_{M}\left(N_{\bar{M}}\right)$ (assumed distinct from the tangent plane to $N_{x}$ at $x$ ) passes through the point $\bar{M}(M)$.

\section{REFERENCES}

1. C. C. Hsiung, $A$ general theory of conjugate nets in projective hyperspace, Trans. Amer. Math. Soc. vol. 70 (1951) pp. 312-322.

2. B. Su, On the surfaces whose Wilczynski quadrics all touch a fixed plane, Revista de la Universidad Nacional de Tucumán (A) vol. 5 (1946) pp. 363-373.

The University OF Kansas 